
\title{
ScIDoC
}

\section{Cutaneous T Cell Lymphoma (CTCL) Superimposed on Disseminated Cutaneous Leishmaniasis (DCL) in an Immunocompromised Female from Yemen}

Al-Kamel M Ahmed ${ }^{1,2^{*}}$

${ }^{1}$ Chair, Regional Leishmaniasis Control Center (RLCC), Yemen.

${ }^{2}$ Sana'a University Hospital, Department of Dermatology, Sana'a, Yemen.

\section{Abstract}

Leishmaniasis and malignancy are emerging public health threats, manifested by a myriad of clinical presentations, and are responsible of substantial morbidity and mortality. Association of cutaneous leishmaniasis (CL) and cutaneous T cell lymphoma (CTCL) is an unusual syndrome that has rarely or even not been reported. Hereby, I report a challenging and an exceedingly rare entity of CTCL superimposed on disseminated CL (DCL) in an immunocompromised woman from Yemen. Hence, many of the questions raised in the context of parasitic carcinogenesis and the supposed potential of Leishmania parasites as oncogenes and/or risk factors for neoplastic development. Understanding this type of relationship between such skin intracellular parasites and carcinogenesis may enrich the provision of prevention, diagnosis and management of these challengeable and very hazardous diseases.

Keywords: Leishmaniasis; DCL; Lymphoma; CTCL; Malignancy.

Abbreviations: CTCL: Cutaneous T Cell Lymphoma; CL: Cutaneous Leishmaniasis; VL: Visceral Leishmaniasis; DCL: Disseminated Cutaneous Leishmaniasis; RLCC: Regional Leishmaniasis Control Center; STAT: Signal Transducers and Activators of Transcription.

\section{Introduction}

Leishmaniasis and malignancy are emerging public health threats manifested by a myriad of clinical presentations and are responsible of substantial morbidity and mortality.

The term "leishmaniasis" refers to a diverse group of syndromes caused by more than 20 species/strains of intracellular, unicellular microorganisms of the genus Leishmania (Trypanosomatidae family) that are transmitted by the bites of their sand fly vectors. Human leishmaniasis can be better classified into two groups of syndromes: (1) stigmatizing and disfiguring cutaneous leishmaniasis (CL), which can affect either the skin (cutaneous form), mucous membranes (mucosal form) or both the skin and cutaneous membranes (mucocutaneous form), and each form can manifest by a myriad of clinical presentations varying from benign, localized nodule/ulcer to extensive disseminated/diffuse CL and post kala- azar dermal leishmaniasis (PKDL); and (2) the potentially lethal if untreated visceral leishmaniasis (VL), which reflects dissemination of Leishmania parasites throughout the reticuloendothelial system. Patients with localized cutaneous leishmaniasis contain the parasite within granulomas, whereas patients with diffuse cutaneous leishmaniasis (DCL) show uncontrolled parasite spread. The cause of this progression remains unknown. However, the alteration of expression levels for genes in immune response signaling pathways could predispose to DCL development and/or be associated with disease severity $[1,2,3]$.

Yemen is endemic for all clinical forms of leishmaniasis, and the disease predominantly affects poor rural women and children. Women with leishmaniasis often present late, depend exclusively on unsafe popular therapies [1, 4].

Lymphoma is the name for many different types of cancer that

*Corresponding Author:

Dr. Mohamed Ahmed Naif Al-Kamel (MD),

Regional Leishmaniasis Control Center, P.O.Box 12692, Sana'a, Yemen.

E-mail: dralkamel@hotmail.com

Received: May 23, 2017

Accepted: July 17, 2017

Published: July 18, 2017

Citation: Al-Kamel M Ahmed (2017) Cutaneous T Cell Lymphoma (CTCL) Superimposed on Disseminated Cutaneous Leishmaniasis (DCL) in an Immunocompromised Female from Yemen. Int J Clin Dermatol Res, S3:002, 4-8. doi: http://dx.doi.org/10.19070/2332-2977-SI03002

Copyright: Al-Kamel M Ahmed ${ }^{\circ}$ 2017. This is an open-access article distributed under the terms of the Creative Commons Attribution License, which permits unrestricted use, distribution and reproduction in any medium, provided the original author and source are credited. 
arise in the lymphocytes (immune cells). There are three types of lymphocytes, B lymphocytes (B cells), T lymphocytes (T cells) and natural killer (NK cells). B lymphocytes make antibodies to fight infection; T lymphocytes have many functions, including helping B lymphocytes; and natural killer cells attack cancer cells and viruses. Lymphoma may arise in any of these types of lymphocytes. Lymphoma is divided into two major categories: Hodgkin lymphoma (HL) and non-Hodgkin lymphoma (NHL). Cutaneous T cell lymphomas (CTCLs), lymphoproliferative malignancies of the $\mathrm{T}$ lymphocytes, are a rare group of NHLs, arise primarily in the skin and have various lesions. CTCL is twice as common in men as in women, with an overall annual incidence of 6:1000,000 [5].

It has been hypothesized that CTCL arises from malignant transformation of activated $\mathrm{T}$ cells in the setting of persistent antigenic stimulation or chronic inflammation [6].

Hereby, I report a challenging and an exceedingly rare entity of CTCL superimposed on DCL in a corticosteroids and antibiotics related immunocompromised patient from Yemen.

\section{Case Report}

\section{First Admission}

In October 2016, a 50-year-old woman from Yemen was referred to Regional Leishmaniasis Control Center's (RLCC) clinic in Sana'a complaining of disfiguring lesions on the face of 6-month duration.

Physical examination revealed: a $2 \mathrm{~cm}$-diameter ulcer on the left tragus (initial lesion); several, firm, painless and erythematous nodules of different sizes ranging from few millimeters to several centimeters in diameter spreading all over her face and sparing mucous membranes, which morphologically resembling lion's face "leonine facies"; some lesions had coalesced to form a giant multi-nodular plaque measuring $>13 \mathrm{~cm}$ in diameter on left cheek; a $3 \mathrm{~cm}$-diameter raised, ulcerated with indurated borders nodule on left buttock area; a $2 \mathrm{~cm}$-diameter raised inflammatory nodule on the left upper back area; mild facial edema (puffiness), oily inflammatory exudation, partial loss of eyebrows and frontal scalp hair, mild itching and a strong sense of discomfort (Figure 1).

History revealed that the first ulcer has developed upon a chemical agent-induced wound on the left cheek, gradually progressed and spread to cover large areas of the face (and elsewhere on the body); patient's inhabitance is a village endemic with leishmaniasis; and a medical history of chronic urticaria of 2-year duration, for which she chronically used oral and parenteral corticosteroids. Patient reported that before admission, she had visited many physicians; subjected to many laboratory investigations; and blindly received numerous medical and non-medical therapies, including chronic oral and parenteral antibiotics, corticosteroids and some popular treatments; however, her condition had been worsen over time. Upon a leishmaniasis-positive slit skin smear specimen, she was sent to our center for further management.

Based on the history, clinical features and skin smear for leishmaniasis report, a presumptive diagnosis of lepromatoid presentation of DCL has been assumed and a differential diagnosis including many infectious and noninfectious diseases, such as LL, CL, chromomycosis, cutaneous sarcoidosis, lymphoma and cutaneous malignancies, has been achieved.

General hematology tests revealed no specific data, skin smears for leishmaniasis and leprosy were negative, and laboratory screening for hepatitis and HIV was negative.

On the second day, the first $4 \mathrm{~mm}$-diameter punch biopsy was excised from the edge of the nodular plaque on left cheek and sent for professional histopathological examination, which later reported (Figure 2): "benign skin with irregular acanthosis; infiltration of the underlying dermis by chronic inflammatory cells; intra- and extra-cellular Leishmania bodies; and no evidence for malignancy or leprosy". Accordingly, a 3-week course of parenteral $600 \mathrm{mg} /$ day sodium stibogluconate (SSG [Pentostam $\left.{ }^{\circledR}\right]$ ), accompanied by oral azithromycin $500 \mathrm{mg}$ /day. However, clinical response was weak. Although the primary ulcers and nodules on the face, back and left buttock area regressed, as well as patient's discomfort, rather improved, there was an evolution of other nodules, which had subsequently progressed, increased in number and size and accompanied with puffing edema and closure of eyes.

\section{First Referral}

In December 2016, the patient was referred to another teaching health institution for further diagnosis where another biopsy was excised and studied and reported the same histological diagnosis.

\section{Second Admission}

In January 2017, patient was referred-back to our center (Figure 3-A, B), and subsequently received an alternative course of oral Itraconazole $200 \mathrm{mg} /$ day, terbinafine $250 \mathrm{mg}$ /day and potassium iodide, without advantage.

\section{Second Referral}

In February 2017, patient was referred abroad to a specialized oncology center in Cairo, Egypt, where the progressively, newly developed lesions (Figure. 3-C) were biopsied and patient underwent further investigations. Histopathological and immunohistochemical reports revealed, dense diffuse atypical lymphoid infiltrate that is diffusely $\mathrm{CD} 3$ positive admixed with large numbers of CD20 positive cells; $T$ cells are CD4 positive and only very sparse cells are CD8 positive; and CD56 is negative. Histopathological and immunohistochemical features provisionally pointed to the tumor stage mycosis fungoides (MF).

CT examination and abdominal sonography revealed, bilateral cervical lymphadenopathy; bilateral axillary lymphadenopathy; abdomino-pelvic lymphadenopathy; mild hepato-splenomegaly; and minimal ascites.

\section{Prognosis}

Accordingly, patient has been receiving systemic cytotoxic chemotherapy regimen. Two months later (until this writing) follow-up has showed a relatively good prognosis (Figure 4). 
Figure 1. Presenting lesions on first admission: (A) pre-auricular ulcer (initial lesion) and big nodular plaque on left cheek; (B) diffuse nodular lesions on right cheek; (C) raised inflammatory nodule of left upper back; and (D) ulcerated nodule with a raised and indurated border (volcano sign) on left buttock area.

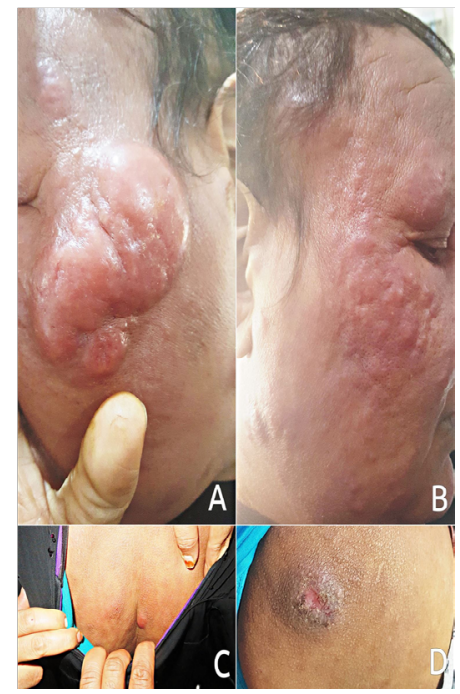

Figure 2. First punch-biopsied tissue showing infiltration of the dermis by chronic inflammatory cells, intra- and extracellular Leishmania bodies ( $\mathrm{H}$ and $\mathrm{E}, \times 100)$.

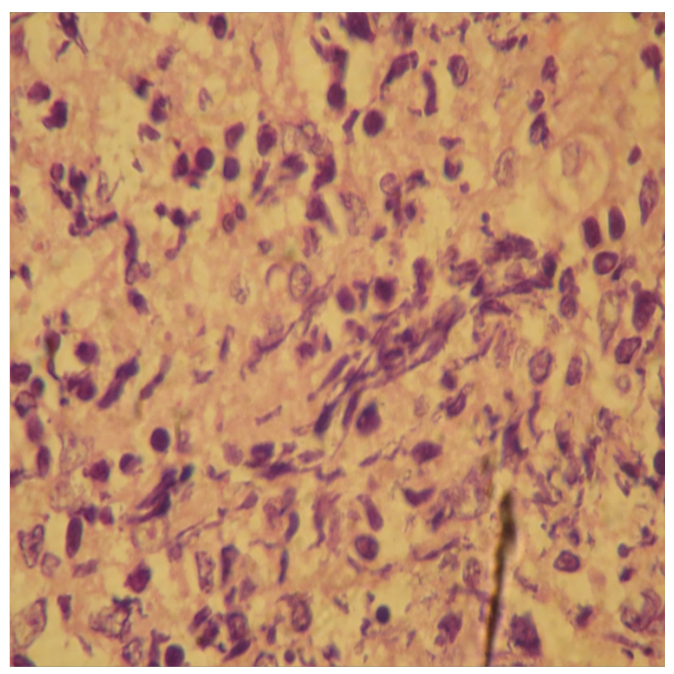

Figure 3. Patient photographs: (A \& B) on the second admission; and (C) rapidly progressing lesions on the face, just before the second referral.

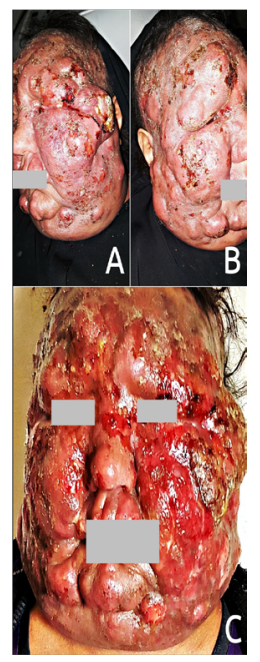


Figure 4. Patient condition after two months of treatment with anti-lymphoma cytotoxic regimen.

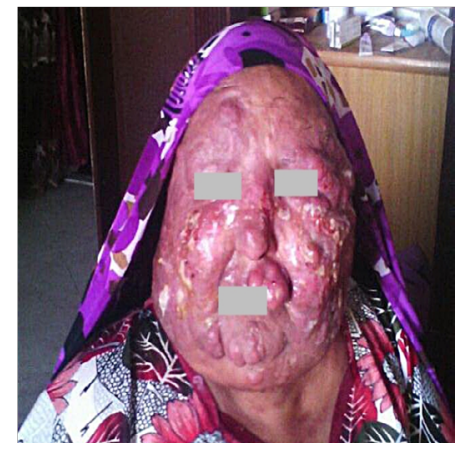

\section{Discussion}

The diverse clinical presentation of CL depends on numerous factors, such as type and duration of clinical lesion, strain of organism, parasitic load, geographic location and host immunocompetence [7].

Diffuse form of CL (DCL) is a common presentation at RLCC, and leprosy has been prevalent in Yemen for long centuries [8, 9].

DCL and Lepromatous Leprosy (LL) are easily confusing and usually develop secondary to an underlying deficiency in cellular immunity. DCL usually present with multiple lesions, difficult to be diagnosed and respond less favorably to treatment. Lepromatoid CL or pseudo-lepromatous leishmaniasis, a rare form of DCL with characteristic spreading of the initial lesion leading to an erythematous and infiltrated plaque, is remarkable for its chronicity and striking resemblance to the skin manifestations of LL and usually caused by L. aethiopica [10-12].

In the current case, history, initial lesions (leishmanial active ulcer on left tragus and volcano sig on left buttock area), clinical features, old slit skin smears for leishmaniasis and diagnostic histological studies had pointed to leishmaniasis (DCL [lepromatoid CL]) as the primary pathology that was masqueraded as LL.

The author supposed that, this patient was bitten by female sandfly at the site of chemical injury on left cheek, acquired Leishmania parasites and leishmaniasis disease start to develop and disseminate; late presentation most probably has played an important role in the dissemination and prognosis of the disease.

Despite a wide therapeutic armamentarium, none has been found to be completely effective and satisfactory and the control of Leishmania infection relies primarily on chemotherapy until date. Organic pentavalent antimonials (Sb5) have been the first-line drugs for the treatment of leishmaniasis for the last six decades, and clinical resistance to these drugs has emerged as a primary obstacle to successful treatment and control [13].

Pentostam ${ }^{\circledR}$ (SSG [Sb5]), freely supplied to patients with leishmaniasis in Yemen through RLCC as part of Stiefel/GSK medicine donation since mid-2014, is the standard chemotherapy for such presentation of CL.

Thereafter, aggressively progression of lesion, characteristic clinical picture, late radiographic, histopathologic and immunohistochemical examinations had provisionally pointed to the tumor stage of mycosis fungoides (MF), the most common form of cutaneous T-cell lymphoma.

The author supposed that, the prior blind long-term antibiotics and corticosteroids courses the patient had been received might have suppressed patient's immunity and masked Leishmania parasites at the initial lesions as per negative subsequent slit smear specimen; the limited response and incomplete resolution of lesions after the initial treatment course with SSG probably indicates a less susceptibility to Sb5 compounds and/or association of secondary pathology occurred concurrently or superimposed on the primary leishmaniasis disease.

Signal transducers and activators of transcription (STAT) proteins have a dual role as cytoplasmic signaling proteins and nuclear transcription factors. Furthermore, STAT signaling plays a central role in mediating inflammation. The STAT family is comprised of seven genes that code for STAT1, STAT2, STAT3, STAT4, STAT5A, STAT5B and STAT6, and most of these STATs as well as their associated signaling mediators have been shown to be critical in immunity and/or pathogenesis of various forms of Leishmania infection. Leishmania are also known to exploit the STAT signaling pathway to favor parasite establishment, growth and replication. Persistent activation of transcription factors of STAT protein family has been implicated in the pathogenesis of a wide variety of human cancers, including CTCL $[14,15]$.

For the present concurrent occurrence of these two diagnosis and treatment challenging clinical entities, leishmaniasis and malignancy, it is speculated that Leishmani infection predispoded to cutaneous $\mathrm{T}$ cell lymphoma development by different know or/ and unknown pathogenic pathways.

Regarding carcinogenic pathogenesis mechanisms in this case, it has been speculated that immunosuppression, the persistent antigen stimulation caused by Leishmania parasites which has induced continuous stimulation of $\mathrm{T}$ cells and chronic inflammation had eventually predisposed to the development of a malignant $\mathrm{T}$ cell clone.

To the author's knowledge, association of cutaneous leishmaniasis and cutaneous T cell lymphoma is an unusual syndrome that has rarely or even not been reported.

Hence, many of the questions raised in the context of parasitic carcinogenesis and the supposed potential of Leishmania parasites as oncogenes and/or risk factors for neoplastic development. 
Understanding this type of relationship between such skin intracellular parasites and carcinogenesis may enrich the provision of prevention, diagnosis and management of these challengeable and very hazardous diseases.

\section{References}

[1]. Al-Kamel MA (2017) Stigmata in cutaneous leishmaniasis: Historical and new evidence-based concepts. Our Dermatol Online. 8(1): 81-90. doi: 10.7241/ourd.20171.21.

[2]. Aronson N, Herwaldt BL, Libman M, Pearson R, Weina P, et al., (2017) Diagnosis and Treatment of Leishmaniasis: Clinical Practice Guidelines by the Infectious Diseases Society of America (IDSA) and the American Society of Tropical Medicine and Hygiene (ASTMH). Am J Trop Med Hyg. 96(1): 24-45. doi:10.4269/ajtmh.16-84256.

[3]. Edith A Fernández-Figueroa, Iván Imaz-Rosshandler, Juan E CastilloFernández, Miranda-Oritz H, Becker I, et al., (2016) Down-Regulation of TLR and JAK/STAT Pathway Genes Is Associated with Diffuse Cutaneous Leishmaniasis: A Gene Expression Analysis in NK Cells from Patients Infected with Leishmania mexicana. PLOS Negl Trop Dis. 10(4): e0004666. doi: 10.1371 /journal.pntd.0004666

[4]. Al-Kamel MA (2016) Impact of leishmaniasis in women: a practical review with an update on my ISD-supported initiative to combat leishmaniasis in Yemen (ELYP). Int J Women's Dermatol. 2(3): 93-101. DOI: http://dx.doi. org/10.1016/j.ijwd.2016.04.003. PMCID: PMC5418880.

[5]. Geskin Larisa J (2014) "Chapter 105. Cutaneous T-cell lymphoma (my- cosis fungoides and Sézary syndrome)" : Williams Hematology. (8th edn), McGraw-Hill Global Education Holdings, LLC, USA.

[6]. Henry K Wong, Anjali Mishra, Timothy Hake, Pierluigi Porcu (2011) Evolving Insights in the Pathogenesis and Therapy of Cutaneous T-cell lymphoma (Mycosis Fungoides and Sezary Syndrome. Br J Haematol. 155(2): 150-66.

[7]. Ul Bari A, Raza N (2010) Lupoid cutaneous leishmaniasis: a report of 16 cases. Indian J Dermatol Venereol Leprol. 76(1): 85.

[8]. Al-Kamel MA (2015) Leishmaniasis in Yemen: A clinico-epidemiological study of leishmaniasis in Central Yemen. Int J Dermatol. 55(8): 849-55.

[9]. Al Qubati Y (2012) Leprosy among migrant workers: ensuring proper treatment. Lepr Rev. 83(4): 335-9.

[10]. Francisco G Bravo, Salim Mohanna (2009) Parasitology. Skin infections: Diagnosis and Treatment. Cambridge: Cambridge University Press, 2009: 117.

[11]. Dassoni F, Abebe Z, Naafs B, Morrone A (2013) Cutaneous and mucocutaneous leishmaniasis resembling borderline-tuberculoid leprosy: a new clinical presentation? Acta Derm Venereo 93(1): 74-7.

[12]. Vandana Mehta, C Balachandran, Raghavendra Rao, Dil SK, Indusri L (2009) Diffuse Cutaneous Leishmaniasis in HIV. Dermatol Online J. 15(4): 9

[13]. Ashutosh, Shyam Sundar, Neena Goyal (2007) Molecular mechanisms of antimony resistance in Leishmania. J Med Microbiol. 56(2): 143-53.

[14]. Steve Oghumu, James Stock, Cesar Terrazzas, Natarajan G, Sanjay V, et al., (2015) Leishmania: Current Biology and Control. U.K: Caister Academic Press, 2015: 107-120.

[15]. Van V, Litvinov BC, Simon Fredholm, Zhou Y, Pehr K, et al., (2014) Analysis of STAT4 expression in cutaneous T-cell lymphoma (CTCL) patients and patient-derived cell lines. Cell Cycle. 13(18): 2975-82. 